\title{
Using Mass Resolving Power as a Performance Metric in the Atom Probe
}

Fred Meisenkothen ${ }^{1}$, Thomas F. Kelly², Edward Oltman², Joseph H. Bunton ${ }^{2}$, Ludovic Renaud ${ }^{3}$, and David J. Larson ${ }^{2}$

${ }^{1}$ National Institute for Standards and Technology, Gaithersburg, MD U.S.A.

${ }^{2}$ CAMECA Instruments, Inc., Madison, WI U.S.A.

${ }^{3}$ CAMECA SA, Gennevilliers, France

The measure of the peak width, $\Delta \mathrm{m}$, in a mass spectrum is one of the most important metrics used to assess mass spectrometer performance. The peak width is often expressed as the mass resolution by normalizing it to the mass of the peak, i.e. $\Delta \mathrm{m} / \mathrm{m}$. The reciprocal of the mass resolution, the mass resolving power (MRP), may also be used. Instruments with a higher mass resolving power can discern individual mass peaks better than instruments with a poorer mass resolving power [1]. The MRP of a spectrometer also impacts the signal-to-noise ratio for a peak and hence the detection limit for a given ion species [2]. To a first approximation, MRP in atom probe tomography will depend on the acquisition voltage (V), the flight path length (L), and the mass-to-charge state ratio $(\mathrm{m} / \mathrm{n})$. The MRP is normally calculated at fixed values of $\mathrm{V}, \mathrm{L}$, and $\mathrm{m} / \mathrm{n}$. For most time-of-flight mass spectrometry techniques, the $(\mathrm{m} / \mathrm{n}), \mathrm{L}$, and $\mathrm{V}$ are constant throughout data acquisition. However, in an atom probe experiment, both the $\mathrm{V}$ and the $\mathrm{L}$ vary throughout the data acquisition process.

A CAMECA LEAP ${ }^{\circledR} 4000 \mathrm{X}-\mathrm{Si}^{\dagger}$ straight-flight-path atom probe instrument was employed in this study. A central flight path length of $90 \mathrm{~mm}$ was chosen for all of the experiments. The analysis chamber pressure was $<2.3 \times 10^{-10}$ Torr. In all, data were collected for thirty-five, [001]-oriented, silicon pre-sharp material (PSM) specimens from five different PSM coupons. Though the laserpulsed mode was employed for most experiments, some voltage-pulsed data were also acquired for comparisons. For convenience, the MRP was defined at full width at half maximum (MRP FWHM $_{\text {) and full width at tenth maximum (MRP }}$ FWTM). The data collection and analyses were accomplished with the CAMECA Data Acquisition and Visualization (DAVis ${ }^{\circledR}$ ) software, version 4.2.1, and the CAMECA Integrated Visualization and Analysis Software (IVAS ${ }^{\circledR}$ ), versions 3.6.6 and 3.6.8, respectively. Seven different local electrodes were represented in the data set.

The results indicate that the MRP can vary by $>29 \%$ between experiments if different acquisition voltage ranges or different detector fields of view, i.e. detector region of interest (ROI) sizes, are used for the analyses. MRP variations of this magnitude are sufficient to obscure the MRP variations that can result from changes to user-defined acquisition parameters, such as specimen base temperature, laser pulse frequency, and laser pulse energy. However, by working within a well-defined voltage range, and at a fixed detector ROI size, it may be possible to calculate MRPs that are meaningful for comparative evaluations. New guidelines for calculating MRP values in the atom probe are proposed based on these experimental findings. Using these new guidelines, the $\mathrm{MRP}_{\mathrm{FWHM}}$ and the $\mathrm{MRP}_{\mathrm{FWTM}}$ for silicon $(<0.01 \Omega \mathrm{cm})$ were found to be relatively insensitive to large changes in three user-defined acquisition conditions, i.e. laser pulse energy, laser pulse 
frequency, and specimen base temperature, thereby making the silicon PSM material a good candidate material for benchmarking instrument performance. Further, given the large number of analyses conducted under similar acquisition conditions, it was possible to generate a statistically significant assessment of the specimen-to-specimen uncertainty for the PSM-based MRP measurements. While the present study was conducted with silicon PSM specimens, similar MRP trends, with respect to $\mathrm{V}$ and detector ROI size, were also observed in amorphized silicon, copper, tungsten, aluminum, and boron specimens. Three new MRP-based performance metrics are also proposed for use in making comparisons between different atom probe instrument designs.
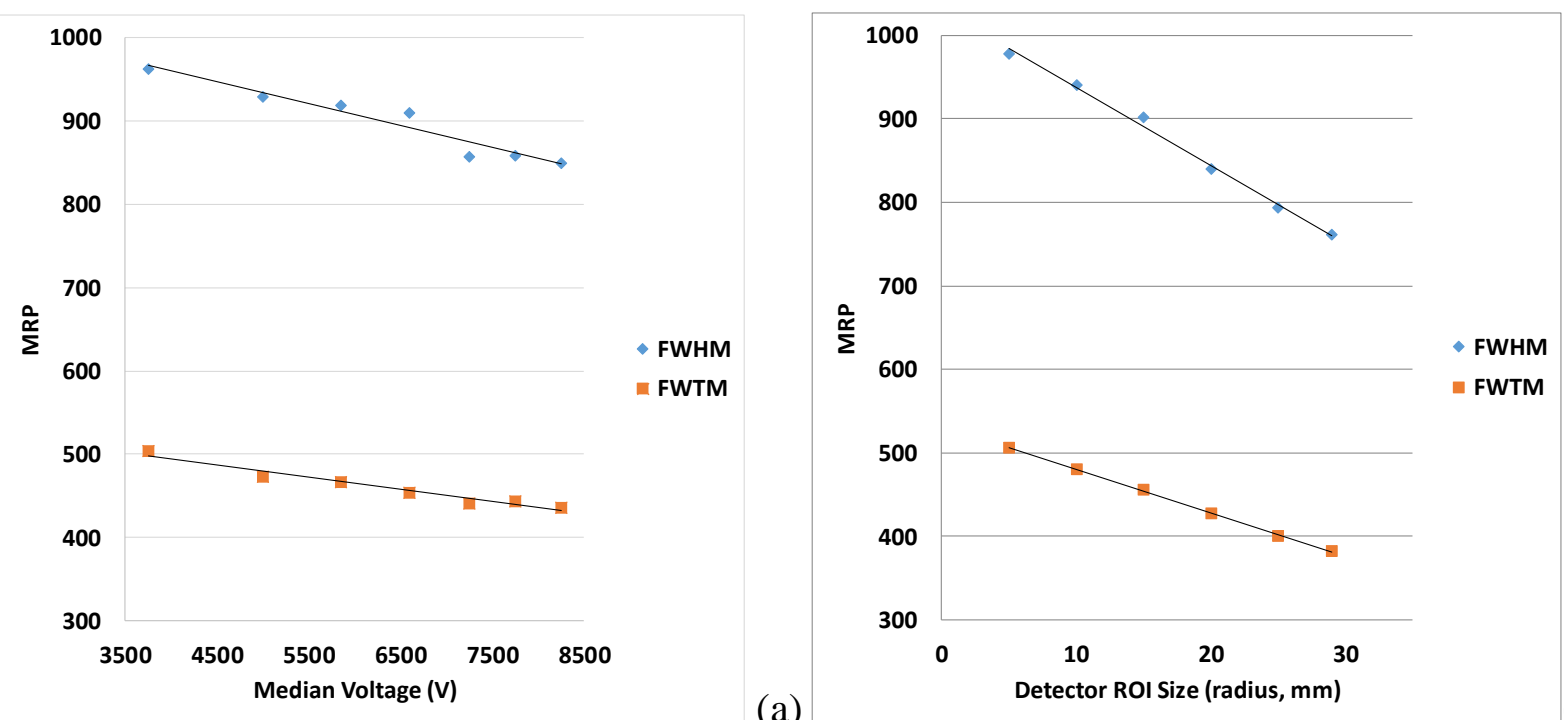

Figure 1: $\mathrm{MRP}_{\mathrm{FWHM}}$ and $M \mathrm{MP}_{\mathrm{FWTM}}$ as a function of (a) median acquisition voltage and (b) detector ROI size, for two different laser-pulsed PSM specimens.

\section{References:}

[1] P.H. Clifton, et al., Microscopy and Microanalysis 14 (2008), p. 454.

[2] L. Currie, Analytical Chemistry 40 (1968) p. 586.

[3] The authors would like to thank Dr. Eric Steel of NIST and Mr. Robert Ulfig, Dr. Ty J. Prosa, and Dr. Hugues Francois Saint Cyr of CAMECA for helpful discussions and encouragement during the course of this work.

†isclaimer:

Certain commercial equipment, instruments, or materials are identified in this paper in order to specify the experimental procedure adequately. Such identification is not intended to imply recommendation or endorsement by the National Institute of Standards and Technology, nor is it intended to imply that the materials or equipment identified are necessarily the best available for the purpose. 